\title{
An analysis of barbiturate-induced eating and drinking in the rat
}

\author{
P. J. WATSON and VERNE C. COX \\ The University of Texas at Arlington, Arlington, Texas 76019
}

\begin{abstract}
Systemic injections of pentobarbital and phenobarbital induced nondeprived animals to eat and to drink. The feeding was not secondary to osmotic changes brought on by drinking, and the drinking was not dependent upon eating. Responses to pentobarbital were quantitatively and qualitatively different from behaviors elicited by lateral hypothalamic stimulation.
\end{abstract}

Subanesthetic doses of hypnotic barbiturates injected systematically influence a number of consummatory behaviors in the rat. Pentobarbital and phenobarbital have potentiated drinking following water deprivation (Knowler \& Ukena, 1973; Schmidt, 1958) and following injection of a hypertonic saline solution (Schmidt \& Dry, 1963a). Drinking potentiation effects also have been discovered with barbital (Schmidt \& Moak, 1959), diallybarbituric acid (O’Kelly \& Weiss, 1955), amobarbital, and secobarbital (Schmidt \& Dry, 1963b). A pentobarbital elicitation of eating in satiated animals and an enhancement of shock-induced aggressive behavior was reported by Jacobs and Farel (1971). Beach (1967) observed a tendency of pentobarbital to facilitate male sexual behavior. Barbiturate potentiation effects also have been demonstrated for the operant responding of food-deprived subjects (e.g., Geller \& Seifter, 1960; Waller \& Morse, 1963). All these findings are consistent with the notion that hypnotic barbiturates can induce or modulate motivation states.

The purpose of this series of experiments was threefold. First, an attempt was made to further explore the motivational effects of pentobarbital and phenobarbital. For example, we wished to determine if there were barbiturate influences on drinking in water-satiated animals as have been reported for eating in food-satiated subjects. A second purpose was to present evidence relevant to possible explanations of the drug actions. When one treatment has such wide-ranging motivational influences, two alternatives seem tenable. The drug may affect the physiological substrates of a number of different motivational systems, or it may affect a single sub-

This research was supported, in part, by the Organized Research Fund of the University of Texas at Arlington. The able assistance of Cynthia Wyatt and M. A. Short is gratefully acknowledged. An earlier version of this paper was presented at the Southwestern Psychological Association Convention, Houston, Texas, April 1975. Reprints may be obtained by sending requests to P. J. Watson, Psychology Department, University of Texas at Arlington, Arlington, Texas 76019. strate common to the various systems. In other words, the injections could be having specific or general motivational effects. The third purpose was relevant to other research conducted in this laboratory on motive states induced by brain stimulation. We sought to determine if there was a relationship between the behavioral states induced by barbiturates and by electrical stimulation of the lateral hypothalamus. Both procedures can affect a broad spectrum of responses, and Jacobs and Farel (1971) have suggested that both manipulations induce a nonspecific arousal state which in turn yields the consummatory behavior.

\section{EXPERIMENT I}

The first experiment was designed to ascertain whether or not systematic injections of sodium pentobarbital could elicit drinking in nonthirsty animals; thus far, as Schmidt (1969) has pointed out, no evidence exists indicating that such a result can be obtained. However, intraventricular application of pentobarbital has evoked drinking in nondeprived subjects (Baile, Scott, \& Mayer, 1967); therefore, reason existed to suspect that a systemic injection would be a sufficient stimulus for water ingestion. This issue was of additional interest because these pentobarbital injections should elicit drinking in satiated animals if they create a motivational state similar to that produced in satiated animals with lateral hypothalamic stimulation.

\section{Method}

Subjects. Animals in this and each subsequent experiment were fully mature male albino rats purchased from the Holtzman Company. All rats, except those in the final experiment, had been used previously in maze learning experiments. Twelve subjects were utilized in the first study.

Apparatus. Two drinking chambers were employed. Each was a $14 \times 14 \times 15 \frac{1}{2}$ in. opaque enclosure with a wire mesh floor and lid. A drinking tube projected approximately 1 in. into the cage from a hole 2 in. above the floor and to the side of one wall. Water was contained in a graduated cylinder which enabled measurement of the amount of drunk within $0.5 \mathrm{ml}$, and a silent solid state counter (BRS/LVE) used in conjunction with a drinko- 
meter registered one count each time a lick at the drinking tube closed the circuit between the floor of the chamber and the drinking tube.

Procedure. Subjects were housed individually in standard laboratory cages measuring approximately $7 \times 7 \times 9 \frac{1}{2} \mathrm{in}$. Purina Lab Chow pellets and water were made available ad lib.

Each animal was randomly assigned to either the Drug or the Saline group. Drug animals were to receive sodium pentobarbital injected intraperitoneally (IP, $9.5 \mathrm{mg} / \mathrm{kg}$ ), while Saline rats were to be injected with an equivalent volume of physiological saline. The experiment was conducted in two phases. During the first phase, subjects were adapted to the procedure. Each rat was removed from its home cage and administered an IP saline control injection. The animal was then placed in a drinking chamber in which only water was available. After $30 \mathrm{~min}$, the subject was removed and returned to its home cage. This adaptation phase lasted 3 days, as did the following testing phase. The only procedural alteration in the three final days was the substitution of pentobarbital injections for saline injections when the six drug animals were treated. Each animal was examined daily at the same time each day.

Three dependent variables were monitored during the adaptation and testing phases: amount of water drunk, number of licks at the drinking tube, and latency to drink. Latency was defined as the time from introduction of the subject into the chamber to the time at which the animal first began to drink as indicated by the joint appearance of air bubbles in the water supply and the rapid registering of licks on the drinkometer.

\section{Results and Discussion}

An analysis of variance for each dependent variable indicated no significant differences between the Drug and the Saline animals during the first 3 baseline days. As illustrated in Figure 1, the drug injections had an immediate influence on all three measures of drinking. Following the first treatment, the pentobarbital subjects drank from 2.0 to $10.5 \mathrm{ml}$ of water while control subject intake ranged from 0 to $0.5 \mathrm{ml}$; similar increments in drinking behavior were obtained for the other two measures. Thus, the Drug animals drank significantly more water $(F=8.44$, $\mathrm{df}=1 / 10, \mathrm{p}<.025)$ and licked significantly more at the drinking tube $(F=11.13, \mathrm{df}=1 / 10, \mathrm{p}<.001)$. In addition, the pentobarbital injections resulted in significantly reduced latencies $(F=24.11, \mathrm{df}=1 / 10$, $\mathrm{p}<.001)$. The Days effect was significant for the number of licks variable $(F=4.46, \mathrm{df}=2 / 20$, $\mathrm{p}<.05)$ but not for the amount drunk $(\mathrm{F}=3.25$, $\mathrm{df}=2 / 20, \mathrm{p}>.05$ ) or for the latency values $(F=.78, \mathrm{df}=2 / 20, \mathrm{p}>.10)$. The Drug by Days interaction was significant for licks $(F=8.52$, $\mathrm{df}=2 / 20, \quad \mathrm{p}<.005)$ and intake $(\mathrm{F}=6.91$, $\mathrm{df}=2 / 20, \mathrm{p}<.005)$, but not latency $(\mathrm{F}=.72$, $\mathrm{df}=2 / 20, \mathrm{p}>.10)$.

The results of this experiment indicate that IP injections of sodium pentobarbital can elicit drinking in water satiated subjects. Two measures, licks and amount drunk, yielded a significant Drug by Days interaction. These outcomes reflect a decreased capacity of the pentobarbital to maintain drinking as a funcion of repeated administration. This may indicate a developing tolerance to the barbiturate,
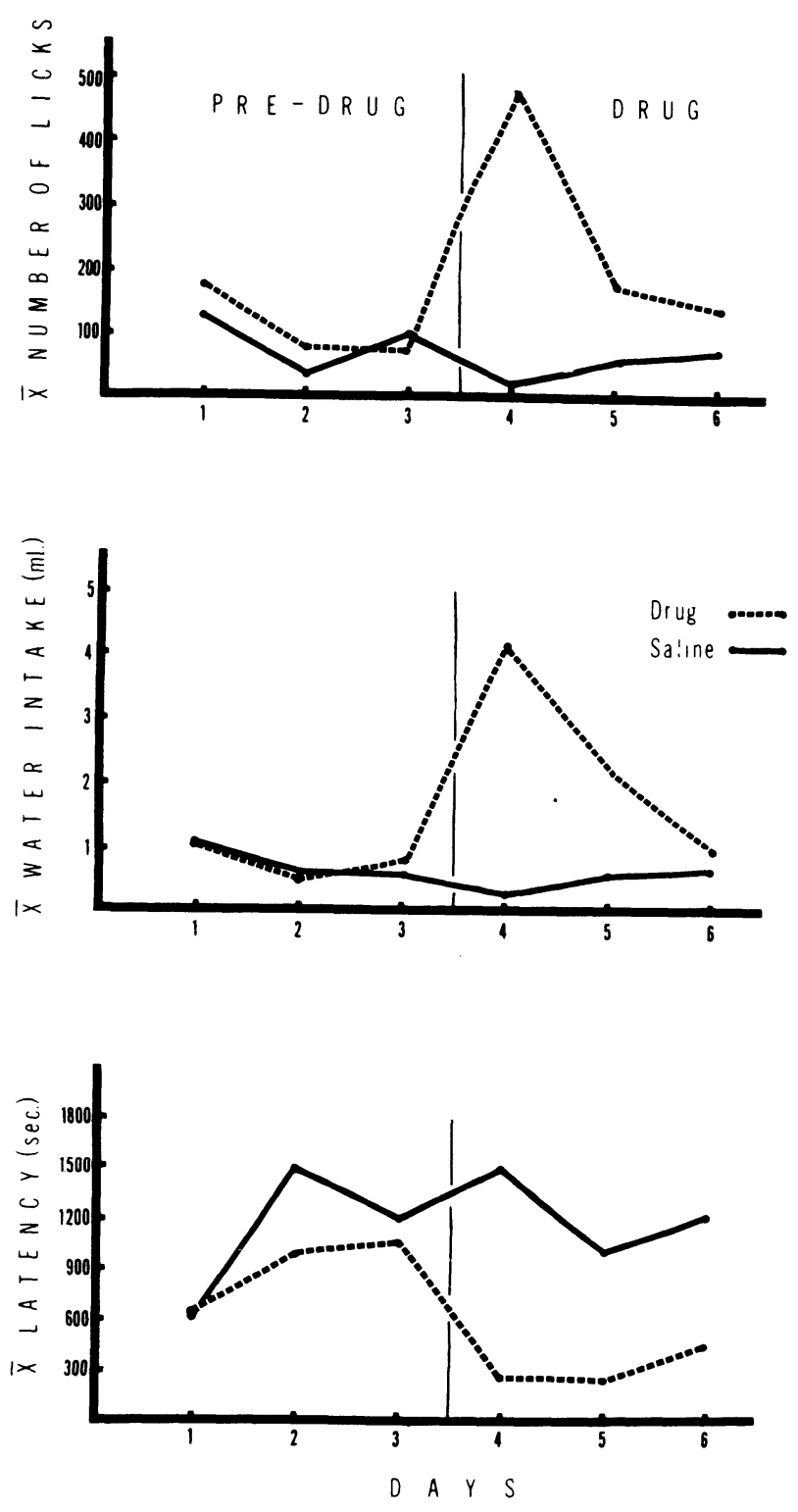

Figure 1. Average measures of water intake before and during sodium pentobarbital treatment for Drug and Saline control subjects.

a phenomenon which has been noticed previously in regards to its anesthetic properties (Singh, Fiegenschue, \& Schexnaydre, 1970) or possibly the habituation of a nonspecific arousal induced by the novelty of the drug state.

\section{EXPERIMENT II}

The finding that pentobarbital can elicit drinking in nonthirsty animals raises questions regarding the determinants of the barbiturate-induced feeding reported by Jacobs and Farel (1971). In that study, animals with ad-lib access to water were given food 30 min after pentobarbital injections. Since it has previously been demonstrated by Kakolewski and 
Deaux (1970) that water ingestion can serve as a stimulus to initiate food intake, the pentobarbital elicitation of eating may not have occurred as a primary drug effect; rather, pentobarbital may have stimulated water intake which in turn could have led to food ingestion. The second experiment evaluated this possibility and in addition, explored possible diurnal influences on the amount of food consumed. Previous research has suggested that phenobarbital injections reorganized diurnal eating patterns (Jones, 1943). Also, the effects of anesthetic and lethal doses of pentobarbital are affected by the time of treatment (Pauly \& Scheving, 1964; Scheving, Vedral, \& Pauly, 1968).

\section{Method}

Subjects. Serving as subjects in this experiment were 48 Holtzman rats.

Apparatus. All testing was conducted in the standard individual housing cage. Two such cages were assigned for each animal with one designated as the home cage and with the other, located just below, designated as the experimental cage.

Procedure. The animals were housed in a room where the lighting cycle was maintained on a $12-\mathrm{h}$ on, 12-h off schedule. Subjects were randomly placed in the Drug-Dark, Drug-Light, Saline-Dark, or Saline-Light group; thus, four groups, each containing 12 rats, were formed.

In the first week, animals were given experience with the diet (Kenl Ration Regular Flavor dog food) used in all adaptation and testing sessions. A fresh supply of this dog food, which supplemented the Purina Lab Chow, was presented daily and was contained in a glass furniture coaster. Water was offered ad lib in the home cage throughout the study. The two phases of the experiment began after this first week. Again, 3 days of adaptation were followed by 3 days of testing; and the dosages of sodium pentobarbital and saline remained unchanged from those utilized in the first experiment. Light animals were treated during the first hour of the light portion of the light/dark cycle, and Dark animals were injected during the first hour of the dark portion.

To help insure that the rats were satiated during experimentation, a fresh supply of food was presented to each subject in his home cage $30 \mathrm{~min}$ before injection. Then the animal was removed from its home cage, given the appropriate injection, and placed in the experimental cage where additional food was available. No water was available while an animal was in the experimental cage. After $30 \mathrm{~min}$, the subject was returned to its home cage, and the amount of food eaten was measured. Care was taken to collect all spillage and to control for evaporation of fluid content in the food.

\section{Results and Discussion}

The Drug and Saline groups did not respond differentially to the predrug control injections, nor was there a time of injection effect observed during this phase. Because of heterogeneity of variance, the data were subjected to a logarithmic transformation $(\log X+1)$ for analysis purposes. Figure 2 demonstrates that both Drug groups ate more than the two control groups $(F=62.15$, df $=1 / 44, p<.001)$. During this drug phase, the time of injection also had a significant influence on food consumption $(F=5.47$,

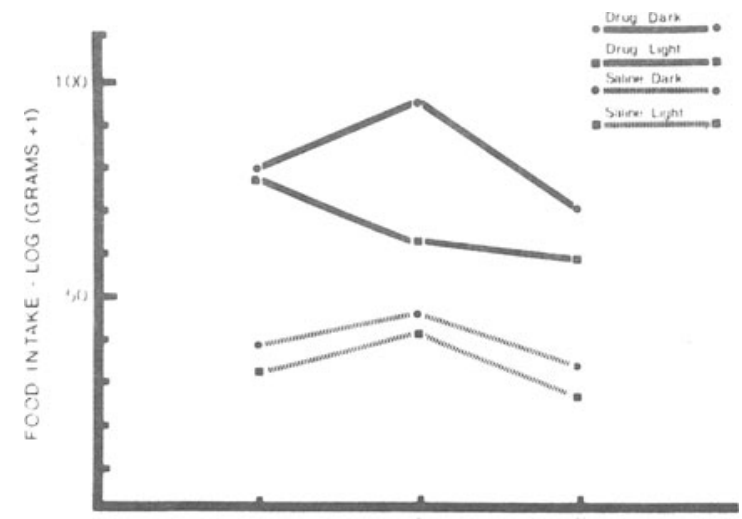

Figure 2. Mean food consumption of animals injected with sodium pentobarbital or physiological saline in the light or in the dark portion of the light/dark cycle. Food intake during drug treatment is presented.

$\mathrm{df}=1 / 44, \mathrm{p}<.05)$, which primarily seemed to reflect the significant Drug by Time by Days effect $(F=5.74$, $\mathrm{df}=2 / 88, \mathrm{p}<.01$ ) observed on Day 2 . The Days effect and all other interactions failed to reach statistically significant levels. In this particular experiment, no major decline in drug-induced food intake was apparent within the 3-day treatment interval; however, extensive observation in our laboratory in the course of other experimentation usually has yielded a decline in eating as rapid as that typically observed for water intake. At any rate, the results of this experiment indicate that pentobarbital-induced eating reflects a direct drug action rather than an effect mediated by water ingestion.

\section{EXPERIMENT III}

The purpose of the third experiment was to establish whether or not sodium phenobarbital has effects which parallel those of sodium pentobarbital. As pointed out previously, phenobarbital, like pentobarbital, potentiates water intake in thirsty animals, and, in fact, there is evidence that its influence is even stronger (Schmidt, 1969).

\section{Method}

Subjects. Twenty-four rats were used in this experiment.

Apparatus. The same drinking chamber and the same homecage experimental-cage arrangement described above were employed.

Procedures. The 24 animals again were housed under a 12 -h on, 12 -h off schedule of illumination. The subjects were randomly divided into two subgroups, one group for examining phenobarbital influences on food intake and one for water intake. Each subgroup in turn was divided into equal-sized Drug and Saline control groups.

The basic procedures for the food and the water sessions outlined above were followed once again with two exceptions. 
First, the phenobarbital injections were administered in $40-\mathrm{mg} / \mathrm{kg}$ dosages, the dosages employed by Schmidt and Dry (1963a) to potentiate water intake in salt-loaded animals. The Saline subjects, as before, were injected with a control volume of physiological saline. Secondly, the possibility of diurnal influences on feeding was not explored; and all subjects in the food-intake experiment were injected during the first hour of the dark portion of the light/dark cycle.

\section{Results and Discussion}

The results of this experiment are reviewed in Table 1, and they indicate that phenobarbital is similar to pentobarbital in its ability to elicit food and water intake in satiated animals. No significant group difference in the amount of food eaten was observed during the predrug condition; however, with the beginning of phenobarbital treatment, the intake of the Drug animals increased to an average of $4.8 \mathrm{~g}$, while controls consumed $1.2 \mathrm{~g}$. Overall, the drug enhancement of eating was statistically reliable $(\mathrm{F}=28.96, \mathrm{df}=1 / 10, \mathrm{p}<.001)$. The Days main effect approached significance $(F=3.38, \mathrm{df}=2 / 20$, $\mathrm{p}=.06$ ), but the Drug by Days interaction did not $(F=2.20, \mathrm{df}=2 / 20, \mathrm{p}>.05)$.

The picture for water intake was similar. All three measures, which did not differ in the predrug situation, immediately reflected a phenobarbitalproduced increase in drinking. The amount of water drunk was greater for the Drug animals than for the Saline $(F=9.90, \mathrm{df}=1 / 10, \mathrm{p}<.025)$, as was the number of licks $(F=19.87, \mathrm{df}=1 / 10, \mathrm{p}<.005)$. The latency values were highly variable; consequently, a log transformation was used. These data revealed a significant reduction in the latency to drink following phenobarbital injection $(F=6.33$, $\mathrm{df}=1 / 10, \mathrm{p}<.05)$. The Days effect did not reach conventional levels of significance for any measure, and the Drug by Days interaction was significant only for the amount measure $(F=5.62, \mathrm{df}=2 / 20$, $\mathrm{p}<.05)$. In conclusion, this experiment established that sodium phenobarbital can cause satiated animals to eat and drink.

\section{EXPERIMENT IV}

The fourth experiment sought to determine if the pentobarbital influences on food and water ingestion have similar latencies. It was thought that different latencies would weaken a simple nonspecific motivation interpretation and would suggest the possibility that the drug selectively activates different physiological substrates at different times.

\section{Method}

Subjects. This experiment was conducted with 18 Holtzman rats.

Apparatus. The apparatus was a clear Plexiglas observation chamber with $11 \frac{1}{2} \times 101 / 2 \times 16$ in. dimensions. When appropriate, drinking tubes were inserted through holes on opposite sides of the chamber approximately 2 in. above the wire mesh floor. Dog food, when made available in the apparatus, was contained in two different glass furniture coasters placed at opposite corners of the chamber.

Procedure. Subjects were housed in individual cages with ad-lib supplies of food and water, and random assignment to either the Food or the Water group was accomplished. Food animals received only dog food in the experimental chamber, while Water animals received only water. On the first 3 days, each animal was removed from his home cage, injected with saline, and placed in the observation chamber where the correct goal object was presented. On the fourth day, all rats were given a $9.5-\mathrm{mg} / \mathrm{kg}$ sodium pentobarbital injection before placement in the apparatus; and the latency to display the target response (either eating or drinking) was obtained. Latency was defined as the time from introduction into the apparatus to initiation of the goal-directed behavior. The sessions during adaptation and during testing lasted $10 \mathrm{~min}$, and any subject failing to respond within this time limit was assigned a score of $600 \mathrm{sec}$. The precautions described above to adapt the Food animals to the dog food diet and to insure satiation during testing were used in this experiment as well.

\section{Results and Discussion}

Figure 3 demonstrates that there was a great deal of overlap between the two groups in the latencies

Table 1

Mean and Standard Error of Measures of Food and Water Intake Before and During Phenobarbital Treatment

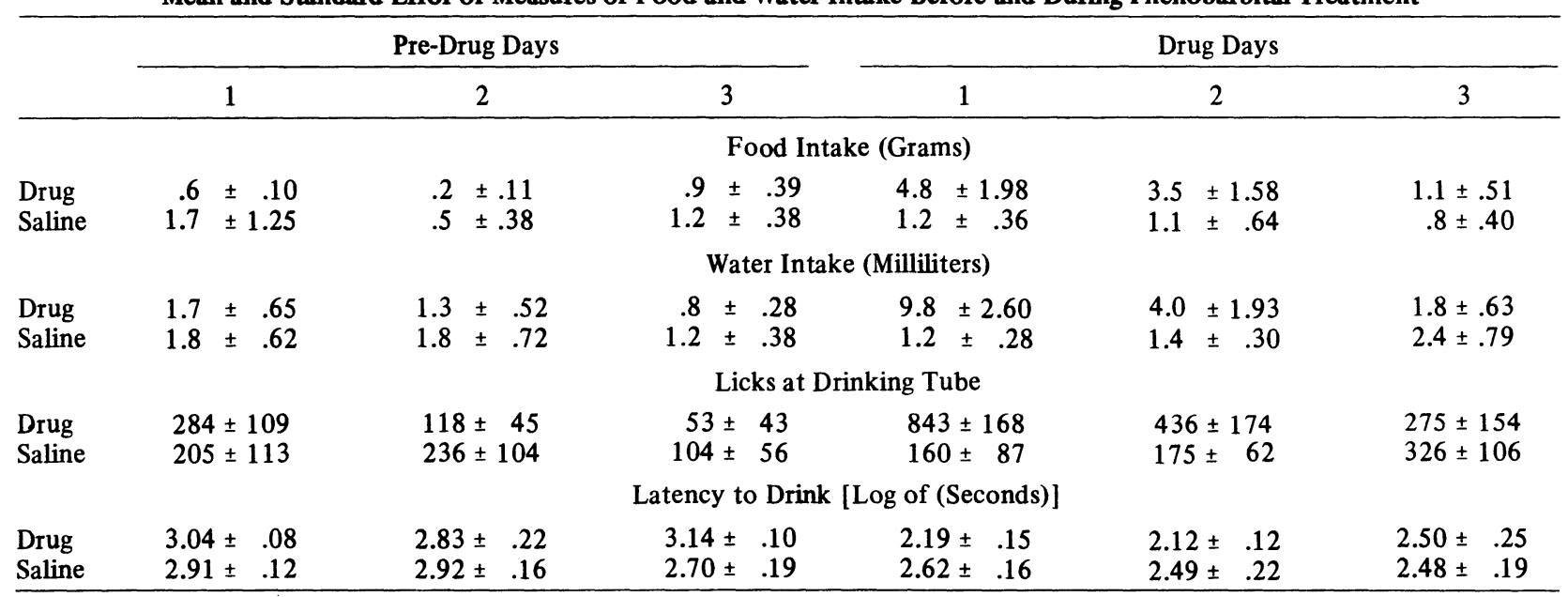




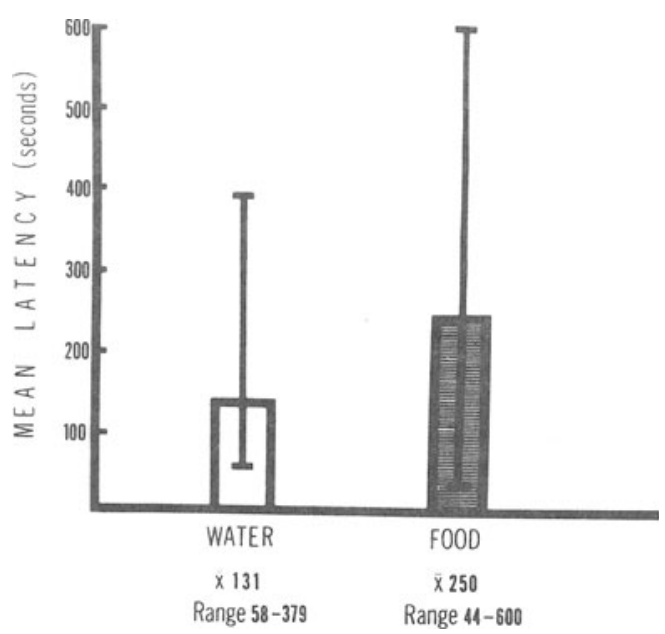

Figure 3. Range and mean latencies of pentobarbital-injected animals to drink or to eat.

obtained. However, the subjects given access to water responded significantly more rapidly than those given food according to a Mann-Whitney $U$ test $(U=12, p<.02)$. In fact, two of the Food animals failed to eat within the 10 -min period, while all the Water animals drank; and the mean latency for the Water subjects was $131 \mathrm{sec}$ compared to $250 \mathrm{sec}$ for the Food subjects. A nonspecific motivation interpretation of barbiturate food and water intake effects would have to accommodate this latency difference.

\section{EXPERIMENT V}

The final experiment directly examined the possibility that a common state underlies behavior elicited by electrical stimulation of the lateral hypothalamus and by barbiturate injection (Jacobs \& Farel, 1971). The findings obtained by Stark and Totty (1967), in which phenobarbital reduced the electrical threshold for stimulation-bound hypothalamic eating, support such a hypothesis. We wished to determine whether an animal's barbiturate response could be predicted based on the nature of his behavior during hypothalamic stimulation. Such a prediction should be possible if, as Jacobs and Farel have argued, a common state underlies barbiturate and brain stimulation-induced behavior.

\section{Method}

Subjects. The animals selected for this study had lateral hypothalamic electrodes which were effective in eliciting one or more behaviors. Each of the 12 rats had a history of extensive stimulation experiences with a number of different goal objects made available both separately and together.

Apparatus. The observation chamber described in the preceding experiment was again utilized. The apparatus was open at the top so that electrode attachment wires could enter from above. Sine-wave stimulation was employed, and programming equipment was used to administer the stimulation and to record the data.

Procedure. Prior to this experiment and before the extensive stimulation experience, the animals were implanted with lateral hypothalamic electrodes (Plastic Products .018-312-.010). The subjects were anesthetized with Equithesin and were positioned in a stereotaxic instrument with the plane on the skull level between bregma and lambda. Coordinates for electrode placement were $3.5 \mathrm{~mm}$ posterior to bregma, $1.6 \mathrm{~mm}$ lateral to the midline, and $8.6 \mathrm{~mm}$ below the surface of the skull.

During this experiment, two goal objects (usually food and water, but food and wood for one subject) were placed in the experimental chamber. Daily stimulation sessions were conducted, and they consisted of 2030 -sec stimulation and interstimulation intervals. Each subject was observed until one of the two behaviors clearly dominated. This response dominance was expressed as the total duration of the dominant response divided by the sum of the durations of both responses; therefore, a score of 1.00 would indicate that only one behavior appeared during the stimulation session. After stable response dominance values were obtained, each animal was observed in a single drug session. On this day, the electrode leads were attached but no stimulation was applied. Instead, the rat received a $9.5-\mathrm{mg} / \mathrm{kg}$ sodium pentobarbital injection and was placed in the chamber for $30 \mathrm{~min}$ with the two goal objects made available and the total duration of each response was noted. When necessary, the drug session was extended until the subject had failed to engage in consummatory activity for $5 \mathrm{~min}$. Another stimulation session was conducted the day following this drug session. For half the subjects, the stimulation session on the day immediately preceding the drug condition was used for comparison purposes, while for the other half, the session on the day following drug treatment was utilized. On these comparison days, each animal received a control saline injection before the stimulation session.

At the end of the experiment, the animals were sacrificed for histological examination of electrode placement. Each subject was given an overdose of anesthetic and then intracardially perfused with $0.9 \%$ saline and $10 \%$ Formalin solutions. Coronal sections were mounted on slides and stained with cresylecht violet.

\section{Results and Discussion}

The behavior of each animal during the two conditions is summarized in Table 2 . It was not possible to predict for all animals the dominance score of a response during the drug condition based on the elicited behavior performance. In fact, there was a nonsignificant correlation $(r=-.10)$ between the dominance score of the dominant stimulation behavior and the dominance score of that behavior during the drug session. A $t$ test for related measures revealed that the dominant stimulation response was significantly weaker during the drug session [ $t(11)=$ $2.427, p<.05]$, with the mean during stimulation equal to .84 compared to .58 during the drug session. This finding cannot be explained by changes in the behavior over time because the statistical analysis included control data from days both before and after the drug session and because the performance of the animals on the day following pentobarbital treatment remained unchanged from that on the day preceding the drug injection $[\mathrm{t}(11)=.413, \mathrm{p}>.50]$. A look at the individual dominance results presents several striking dissociations. For example, subjects $48 \mathrm{~F}$ and $29 \mathrm{HB}$ never drank in any stimulation 
Table 2

Dominance Score (DS) ${ }^{\mathbf{a}}$ of Behavior Elicited by Lateral Hypothalamic Stimulation and by Pentobarbital Injection

\begin{tabular}{|c|c|c|c|c|c|c|}
\hline \multirow[b]{2}{*}{ Subject } & \multicolumn{3}{|c|}{ Stimulation Behavior } & \multicolumn{3}{|c|}{ Pentobarbital Behavior } \\
\hline & $\begin{array}{c}\text { Dominant } \\
\text { Response }\end{array}$ & DS & $\begin{array}{c}\text { First } \\
\text { Response }\end{array}$ & $\begin{array}{l}\text { Dominant } \\
\text { Response }\end{array}$ & DS & $\begin{array}{c}\text { First } \\
\text { Response }\end{array}$ \\
\hline $48 \mathrm{~F}$ & Eating & 1.00 & Eating** & Drinking & 1.00 & Drinking \\
\hline $2 \mathrm{G}$ & Eating & 1.00 & Eating** & Drinking & 1.00 & Drinking \\
\hline $58 \mathrm{G}$ & Eating & .52 & Drinking & Drinking & .504 & Drinking \\
\hline $65 G$ & Eating & .85 & Drinking & Eating & .80 & Drinking \\
\hline $21 \mathrm{H}$ & Eating* & .76 & Drinking** & Drinking & .85 & Drinking \\
\hline $29 \mathrm{HB}$ & Eating & 1.00 & Eating** & Eating & .78 & Eating \\
\hline $2 U^{b}$ & Eating & .91 & Eating & Eating & .88 & Gnawing \\
\hline $3 U$ & Eating* & .93 & Eating & Eating & .95 & Eating \\
\hline $4 U$ & Eating* & .72 & Eating** & Eating & .79 & Drinking \\
\hline $5 U$ & Eating & .64 & Drinking & Eating & .59 & Drinking \\
\hline $8 U$ & Eating & .93 & Eating & Eating & .72 & Drinking \\
\hline $11 \mathrm{U}$ & Eating & .87 & Drinking & Eating & .74 & Drinking \\
\hline
\end{tabular}

${ }^{2} D S=\frac{\text { Duration of dominant response }}{\text { Total duration of both responses }}$

$b_{2} U$ was the only animal to have wood and food available during testing; all other subjects were given access to food and water.

*This animal displayed unusual stimulation-induced responses which were not directed towards the two available goal objects.

**This first response in the single stimulation comparison session was always the first behavior to appear during stimulation sessions in which both of the goal objects were present.

session, while both drank in the pentobarbital condition; and for $48 \mathrm{~F}$, drinking was the only drug response. Subject $2 \mathrm{G}$ only very rarely drank during hypothalamic stimulation, and he was like $48 \mathrm{~F}$ in being a pure eater in the stimulation control session and a pure drinker in the drug session. A strong switch from eating to drinking also was evident for $21 \mathrm{H}$, while $58 \mathrm{G}$ displayed the same change, but of a smaller magnitude.

Other characteristics of the data disclosed contrasts between the hypothalamic and the pentobarbital conditions. For the animals as a whole, hypothalamic stimulation did not consistently elicit eating or drinking as the first behavior; however, the latency results of the fourth experiment suggested that the initial pentobarbital response should have been drinking. This proved to be the case with a mean latency to drink of $295 \mathrm{sec}$ and to eat of $850 \mathrm{sec}$, a statistically significant difference $[\mathrm{t}(10)=2.625, \mathrm{p}<.05]$. On an individual level, three of the five subjects that did consistently display one response or the other as the initial stimulation behavior had a different first response during the drug condition. Finally, unusual responses that occurred during the electrical stimulation never appeared after pentobarbital injection. These included vigorous licking along the floor, licking along the walls, and a characteristic shuffling of the forepaws on the chamber floor. Thus, both quantitative and qualitative aspects of the evidence indicated that the motivational states generated by the two procedures differed substantially; and this weakens the argument that a common process underlies the induction of feeding and drinking by barbiturates and by hypothalamic stimulation. Another possibility deserving consideration is that repeated drug experience might also yield a similar response dominance pattern and that initial stimulation behavior was similar to initial drug behavior. Conclusions based on the asymptotic stimulation data therefore would be strengthened if the data obtained after extended stimulation experience were representative of elicited responding from the outset. We tested the hypothesis that eating was also the stronger behavior during the first stimulation session in which behavior began to appear by comparing the number of trials out of 20 in which eating occurred with the number of trials of drinking. For the 11 animals that eventually displayed both eating and drinking, the average number of eating trials was 11.54 compared to 4.00 trials of drinking, a statistically reliable result $[\mathrm{t}(10)=1.89, \mathrm{p}<.05$, one-tailed test]. Therefore, this suggests that the behavior elicited during initial experience with hypothalamic stimulation was different from the predominant drinking elicited by initial pentobarbital injection.

Histological examination revealed that the electrode tips fell within the boundaries of the lateral hypothalamic area and medial forebrain bundle. Localization of the electrode in subject $58 \mathrm{G}$ was not possible. There was no apparent relationship between electrode location and relative response dominance during stimulation.

\section{GENERAL DISCUSSION}

Systemic injections of both pentobarbital and phenobarbital caused water-satiated animals to drink and food-satiated animals to eat. The feeding was not secondary to osmotic alterations brought on by drinking, and the drinking was not dependent 
upon eating. Water intake occurred sooner after pentobarbital injection than did food ingestion, and this was true for both between- and within-animal observations.

The data present difficulties for a nonspecific arousal or general motivation interpretation of these barbiturate influences. As pointed out previously, the discovery of different latencies for pentobarbitalproduced feeding and drinking makes more plausible the idea that the drug influences two different systems. Even if barbiturate effects were due to the induction of a nonspecific motivational state, the present results raise questions regarding the similarity of such a state to that induced by hypothalamic stimulation which yields feeding and drinking. Our findings indicate that responses to lateral hypothalamic stimulation were quantitatively and qualitatively different from behaviors elicited by barbiturates.

These studies do not offer any clues as to how barbiturates cause food and water intake. The most obvious candidate for the production of drinking would seem to be the sodium content of the injections; however, this explanation seems inadequate for several reasons. First, the amounts of sodium were quite small. Secondly, with the hypnotic barbiturates that have potentiated drinking, the degree of potentiation has not been directly related to the sodium content of the injection (Schmidt \& Moak, 1959). Finally, according to Schmidt (1969), rats injected with sodium phenobarbital and with a hypertonic saline solution did not have serum sodium levels different from animals given only the hypertonic saline, although they drank considerably more.

In reviewing the complex potentiation influences of these drugs on drinking, Schmidt (1964) suggested that for pentobarbital and to some extent for phenobarbital, the effectiveness of the injection represents its ability to attenuate the satiation process. Consistent with this suggestion was the finding that pentobarbital failed to reduce drinking latencies in salt-loaded rats while increasing the total amount of water consumed; therefore, changes in response initiation processes seemed less important than alterations in response termination processes in producing barbiturate drinking effects (Schmidt \& Dry, 1963a). However, animals in the studies reported here, which were not water deprived nor injected with hypertonic saline, displayed both decreased response latencies and increased water consumption. These data argue against an interpretation solely in terms of diminished satiation effects. If response initiation and satiety are mediated by different systems, the present data indicate that both may be affected by barbiturates. It is possible that previous failures to observe drug-induced changes in response latency reflect the fact that in deprived or salt-loaded animals the drinking latency is already at minimal values.
Results reported by Mogenson, McLachlan, Wishart, and Stevenson (1969) raise the possibility that barbiturate effects on drinking and feeding may be mediated by the ventromedial area of the hypothalamus (VMH). They found that VMH lesions prevented the phenobarbital facilitation of drinking, and they claimed that this result supported the satiety reduction hypothesis. Several observations in addition to the findings of Mogenson et al. indicate that the VMH should be examined as a possible substrate for the barbiturate motivational actions; however, its participation may be best conceptualized in terms of alterations in insulin secretion rather than in terms of some hypothetical satiety function. Kulkosky, Porte, and Woods (1975) recently found that intrathecal application of pentobarbital elevated plasma insulin levels, and they concluded that the drug functionally lesioned tissue in the $\mathrm{VMH}$ area that normally inhibits pancreatic activity. Since insulin can elicit both food (Booth \& Brookover, 1968) and water (Booth \& Pitt, 1968; Novin, 1964) intake, the possibility exists that barbiturate-elicited feeding and/or drinking reflects increases in insulin secretion brought on by VMH inactivation. Wagner and DeGroot (1963) reported that pentobarbital injected directly into the VMH potentiated and elicited ingestion of a liquid food diet in rats, and their findings obviously strengthen the argument for VMH involvement. The experimental question remaining to be tested is whether or not nondeprived diabetic or VMH-lesioned rats will respond to systemic barbiturate administration with eating and/or drinking.

Certainly other alternatives merit exploration as well. For example, Mogenson et al. also reported that septal lesions eliminated the phenobarbital drinking influence, implicating this structure as well. In addition, it has previously been shown that pentobarbital reduces body temperature (Lomax, 1966); and since loss of body heat can evoke eating (Brobeck, 1948), barbiturate-induced thermal effects may underlie the elicitation of food consumption. Thus, explanations other than a VMH-insulin interaction deserve consideration.

\section{REFERENCES}

Baile, C. A., Scott, F. A.. \& Mayer, J. Eating and drinking induced by cerebral-ventricle injections in rats with lesions in the lateral hypothalamus. Experientia, 1967, 23, 1033-1034.

BEACH, F. A. Cerebral and hormonal control of reflexive mechanisms involved in copulatory behavior. Physiological Review, 1967, 47, 289-316.

Booth, D. A., \& Brookover, T. Hunger elicited in the rat by a single injection of bovine crystalline insulin. Physiology and Behavior, 1968, 3, 439-446.

Booth, D. A., \& PIтt, E. The role of glucose in insulininduced feeding and drinking. Physiology and Behavior, $1968,3,447-453$. 
Brobeck, J. R. Food intake as a mechanism of temperature regulation. Yale Journal of Biology and Medicine, 1948, 20, 545-552.

Geller, I., \& Seifter, J. The effects of meprobamate, barbiturates, d-amphetamine, and promazine on experimentally induced conflict in the rat. Psychopharmacologia, $1960,1,482-492$.

JACOBS, B. L., \& Farel, P. B. Motivated behaviors produced by increased arousal in the presence of goal objects. Physiology and Behavior, 1971, 6, 473-476.

JoNES, M. R. The effect of phenobarbital on food and water intake, activity level, and weight gain. Journal of Comparative and Physiological Psychology, 1943, 35, 1-10.

KAKolewskI, J. W., \& DeAUX, E. Initiation of eating as a function of ingestion of hypoosmotic solutions. American Journal of Physiology, 1970, 218, 590-595.

KNOWLER, W. C., \& UKenA, T. E. The effects of chlorpromizine, pentobarbital, chlordiazepoxide and d-amphetamine on rates of licking in the rat. Journal of Pharmacology and Experimental Therapeutics, 1973, 184, 385-397.

Kulkosky, P. J., Porte, D., JR., \& Woods, S. C. Elevation of rat plasma insulin by intrathecal pentobarbital. Experientia, 1975, 31, 123-124.

Lomax, P. The hypothermic effect of pentobarbital in the rat: Sites and mechanisms of action. Brain Research, 1966, 1, 296-302.

Mogenson, G. J., McLachlan, R., Wishart, T., \& Stevenson, J. A. F. Effects of sodium phenobarbital on water intake of rats with lesions of ventromedial hypothalamus or septum. Federation Proceedings, 1969, 28, 648. (Abstract).

Novis, D. The effects of insulin on water intake in the rat. In M. J. Wayner (Ed.), Thirst. New York: Macmillan Company, 1964.

O'Kelly, L. I., \& Weiss, H. H. The effects of ether and/a barbiturate on water regulation in the rat. Journal of Comparative and Physiological Psychology, 1955, 48, 123-125.

Pauly, J. E., \& Scheving, L. E. Temporal variations in the susceptibility of white rats to pentobarbital and tremorine. International Journal of Neuropharmacology, 1964, 3, 651-658.
Scheving, L. E., Vedral, D. F., \& Pauly, J. E. A circadian susceptibility rhythm in rats to pentobarbital sodium. Anatomical Record, 1968, 160, 741-750.

Schmidt, H., JR. Pentobarbital facilitation of water ingestion in the albino rat. Journal of Comparative and Physiological Psychology, 1958, 51, 26-28.

Schmidt, H., JR. Water intake as an index of drug action. In M. J. Wayner (Ed.), Thirst. New York: Macmillan Company, 1964.

SchmidT, H., JR. Alterations in central thirst mechanisms by drugs. Annals of the New York Academy of Science, 1969, 157, 962-976.

SCHMIDT, H., JR., \& DRY, L. Comparison of phenobarbital and pentobarbital actions upon water ingestion. Journal of Comparative and Physiological Psychology, 1963, 56, 179-182. (a)

Schmidt, H., JR., \& DRY, L. Dose and time as variables in barbituate action upon water ingestion. American Journal of Physiology, 1963, 204, 817-820. (b)

Schmidt, H., JR., \& MoAk, S. J. Some drug effects influencing barbituate facilitation of water ingestion. American Journal of Physiology, 1959, 196, 307-310.

Singh, J. M., Fiegenschue, B., \& Schexnaydre, C. Development of tolerance to pentobarbital. Journal of Pharmaceutical Science, 1970, 59, 1020-1022.

StaRK, P., \& TotTY, W. Effects of amphetamines on eating elicited by hypothalamic stimulation. Journal of Pharmacology and Experimental Therapeutics, 1967, 158, 272-278.

WAGNER, J. W., \& DEGroot, J. Changes in feeding behavior after intracerebral injections in the rat. American Journal of Physiology, 1963, 204, 483-487.

WALlER, M. B., \& MorSE, W. H. Effects of pentobarbital on fixed-ratio reinforcement. Journal of the Experimental Analysis of Behavior, 1963, 6, 125-130.

(Received for publication February 3, 1976; accepted March 29, 1976.) 